\title{
Demographic Characteristics and Treatment
} Patterns Among Patients Receiving Palbociclib for HR+/HER2 - Advanced Breast Cancer: A Nationwide Real-World Experience

\author{
Katalin Boér (D) \\ Gábor Rubovszky ${ }^{2,3}$ \\ György Rokszin ${ }^{4}$ \\ Zsolt Abonyi-Tóth ${ }^{4,5}$ \\ Csenge Földesi ${ }^{6}$ \\ Magdolna Dank ${ }^{3}$ \\ 'Department of Medical Oncology, Szent \\ Margit Hospital, Budapest, Hungary; \\ ${ }^{2}$ Chemotherapy Department B, National \\ Institute of Oncology, Budapest, Hungary; \\ ${ }^{3}$ Department of Internal Medicine and \\ Oncology, Semmelweis University, \\ Faculty of Medicine, Budapest, Hungary; \\ ${ }^{4}$ RxTarget Ltd., Szolnok, Hungary; \\ ${ }^{5}$ University of Veterinary Medicine, \\ Budapest, Hungary; ${ }^{6}$ Pfizer Ltd., \\ Budapest, Hungary
}

\begin{abstract}
Background: This nationwide retrospective study reports data on the real-world use of the selective cyclin-dependent kinase 4 and $6(\mathrm{CDK} 4 / 6)$ inhibitor palbociclib in a large population of advanced breast cancer $(\mathrm{ABC})$ patients during a 2-year period in Hungary.

Methods: All patients with $\mathrm{ABC}$ who received palbociclib between May 1, 2017 and June 30, 2019 were included in the analysis. Patient demographic and clinical characteristics, disease-related factors and treatment patterns were examined during the early access program and in the regular reimbursement period.

Results: Altogether, 962 patients were included (mean age: 60.6 years). A total of 399 patients $(41 \%)$ were treated with palbociclib plus aromatase inhibitors $(\mathrm{P}+\mathrm{AI})$, and 563 patients $(59 \%)$ received palbociclib and fulvestrant $(\mathrm{P}+\mathrm{F})$. The most commonly prescribed AI was letrozole $(n=359 ; 90 \%)$. Of those with metastatic disease $(n=733 ; 76 \%), 241$ patients (33\%) had visceral metastases and 449 (61\%) had bone-only disease. The majority of patients $(79 \%)$ received palbociclib as first- or second-line therapy for ABC. The starting dose of palbociclib was $125 \mathrm{mg}$ in $98 \%$ of patients; dose reductions were required in $32 \%$ of patients receiving $\mathrm{P}+\mathrm{AI}$ and $31 \%$ of those treated with $\mathrm{P}+\mathrm{F}$. At the time of data collection, palbociclib therapy was ongoing in 270 patients $(68 \%)$ in the $\mathrm{P}+\mathrm{AI}$ group and 245 patients (44\%) in the $\mathrm{P}+\mathrm{F}$ group.

Conclusions: This nationwide analysis is the first to provide insights into the real-world use of palbociclib in a large patient population from a Central-Eastern European country. The findings confirm the good tolerability of palbociclib with similar dose reduction rates to those reported from registration trials.
\end{abstract}

Keywords: palbociclib, metastatic breast cancer, real-world, retrospective

\section{Introduction}

Hormone receptor-positive ( $\mathrm{HR}+) \mathrm{BC}$ is the most common type of $\mathrm{BC}$, and an estimated $60-75 \%$ of advanced $\mathrm{BC}$ (ABC) patients (locally advanced BC and metastatic BC) have HR+/HER2- disease. ${ }^{1-3}$ For decades, the mainstay of treatment for $\mathrm{HR}+\mathrm{ABC}$ was endocrine monotherapy with selective estrogen receptor modulators (eg tamoxifen), selective estrogen receptor downregulators (eg fulvestrant) or a third-generation aromatase inhibitor (AI; eg anastrozole, letrozole or exemestane). ${ }^{4}$ However, the efficacy of endocrine therapy might be limited by the development of endocrine resistance, which affects a significant proportion of
Correspondence: Magdolna Dank Department of Internal Medicine and Oncology, Semmelweis University, Faculty of Medicine, Tömő Utca 25-29, Budapest, I083, Hungary

Email dank.magdolna@med.semmelweisuniv.hu 
patients encountered in clinical practice. ${ }^{5,6}$ The current guidelines of the European Society of Oncology (ESO) and European Society for Medical Oncology (ESMO) define primary endocrine resistance as a relapse within the first 2 years of adjuvant endocrine therapy or disease progression within the first 6 months of first-line endocrine therapy for $\mathrm{ABC}$, while secondary endocrine resistance is defined as a relapse while on adjuvant endocrine but after the first 2 years, or a relapse within 12 months of completing adjuvant ET, or disease progression 6 or more months after initiating endocrine therapy for MBC, while on endocrine therapy. ${ }^{7}$

The development of CDK4/6 inhibitors has revolutionized the treatment landscape for $\mathrm{ABC} .{ }^{8}$ Currently, three CDK4/6 inhibitors (palbociclib, ribociclib and abemaciclib) are approved for the treatment of HR+/HER2metastatic BC which have been shown to provide significant progression-free survival (PFS) benefits when added to endocrine therapy. ${ }^{9}$ Furthermore, the addition of ribociclib or abemaciclib to endocrine therapy significantly improved overall survival (OS) both in the aromatase inhibitor (AI) sensitive and AI resistant settings. ${ }^{9}$ Palbociclib is an orally available, selective CDK4/6 inhibitor which demonstrated significant improvements in PFS when added to letrozole or fulvestrant in patients with ABC in the PALOMA studies both in the endocrine sensitive and resistant settings. ${ }^{10-14}$ In November 2016, palbociclib was registered by the European Medicines Agency (EMA) for the treatment of HR+/HER2- locally advanced or metastatic $\mathrm{BC}$ in combination with an $\mathrm{AI}$, or in combination with fulvestrant in women who have received prior endocrine therapy. ${ }^{15}$ The recommended starting dose of palbociclib is $125 \mathrm{mg}$ once daily for 21 consecutive days followed by 7 days off treatment to comprise a complete cycle of 28 days. Palbociclib became available in Hungary in May 2017 first in the form of named-patient-based reimbursement (NPBR), which provides early access to innovative drugs under the principles of equity. Individual applications are ruled within 60 days of submission and individual reimbursement is generally granted for 3 months, after which the application can be resubmitted regularly as long as the requested drug is effective and tolerable in the particular patient. In October 2018, palbociclib became available under regular reimbursement in Hungary, providing general access to all eligible patients without having to go through the NPBR process. ${ }^{16}$

Although randomized controlled trials (RCT) are the gold standard for demonstrating the efficacy and safety of a new drug, patient populations included in these studies usually do not represent the wide range of patients treated in real-world clinical practice due to the strict inclusion criteria and trial protocols. Indeed, a number of real-world studies have demonstrated clinically relevant differences between RCT populations and real-world BC patients with regard to age, tumor size, visceral metastases, or the percentage of HR-positive BC. ${ }^{17,18}$ Therefore, real-world studies are crucial for examining the impact of new therapies in real-world patient populations, particularly those not represented in clinical trials, as well as for aiding physicians during the treatment of real-life, unselected patient populations. Once introducing new therapies in oncology, the reproducibility of RCT data in real-world populations is an increasing concern, with real-world evidence having an increasing role in health care decisions. ${ }^{19}$

Therefore, the aim of this retrospective study was to describe patient demographics, disease characteristics, and treatment patterns in a population-based sample of patients with HR+/HER2 - ABC treated with palbociclib in combination with endocrine therapy in accordance with the EMA label. Furthermore, we also intended to analyze the advantages of early access to innovative pharmaceuticals.

\section{Methods}

This nationwide, retrospective analysis included patients with $\mathrm{ABC}$ who received at least one dose of palbociclib between May 1, 2017 and June 30, 2019 using data from the National Health Insurance Fund (NHIF) of Hungary. The NHIF covers the whole Hungarian population and contains patient identifiers and International Classification of Disease 10 (ICD-10) code records about all in- and out-patient visits and all prescriptions of reimbursed drugs. Therefore, the NHIF represents a comprehensive data source of all Hungarian patients who received palbociclib therapy.

All prescription claims, in- and out-patient treatment data (diagnostic workup and interventions) of included patients were queried irrespective of their association with $\mathrm{BC}$. Male patients and those receiving any treatment for $\mathrm{ABC}$ other than bisphosphonates, $\mathrm{AI}$ or fulvestrant while on palbociclib treatment were excluded from the analysis. The following demographic and clinical parameters were examined at baseline: 1) age; 2) endocrine partner for palbociclib (AI or fulvestrant); 3) menopausal status; 4) disease stage (locally advanced or metastatic) at diagnosis and type of metastatic disease (de novo or recurrent metastatic); 5) sites of metastases; 6) prior therapy for 
eBC; 7) line of treatment at palbociclib initiation; 8) prior therapy for $\mathrm{ABC}$ before palbociclib initiation.

Pre- or perimenopausal women were defined as receiving GnRH agonist therapy during palbociclib treatment, and the rest of the patients were classified as postmenopausal. Metastatic BC was defined as follows: 1) inpatient occurrence of the ICD-10 code C78 (Secondary malignant neoplasm of respiratory and digestive organs) or C79 (Secondary malignant neoplasm of other and unspecified sites) between diagnosis and the first prescription claim for palbociclib; or 2) radiotherapy administered for metastases; or 3) initiation of bisphosphonate or denosumab treatment before or within 60 days after the initiation of palbociclib therapy. In all other cases, patients were classified as having "Locally advanced" disease.

Visceral metastases were identified based on the occurrence of corresponding ICD-10 codes for liver, lung/ pleura, central nervous system, or ovarian metastases. Patients were considered to have other metastases in the absence of visceral metastases if they had secondary malignant neoplasm of the skin, digestive or urinary organs, retroperitoneum and peritoneum, adrenal gland, or other specified sites. Bone-only metastases were defined based on the presence of bone metastases and the absence of visceral and other metastases, and/or the initiation of bisphosphonate or denosumab therapy before or within 60 days after palbociclib treatment initiation.

De novo metastatic $\mathrm{BC}$ was defined based on the initiation of palbociclib combination therapy within 90 days after diagnosis if the patient received no prior treatments for $\mathrm{ABC}$ apart from the combination partner of palbociclib (AI or fulvestrant) or, the rest of the cases were classified as "Recurrent" breast cancer. Prior treatments for advanced or metastatic BC were investigated among patients who received palbociclib in second or further lines of treatment. The starting dose of palbociclib was also determined $(125 \mathrm{mg} /$ day, $100 \mathrm{mg} /$ day, or $75 \mathrm{mg} /$ day), as well as dose reductions.

Patients were classified into the following groups based on the outcomes and the treatments received at the time of data collection (30 June 2019): 1) ongoing palbociclib treatment; 2) switch to another monotherapy in the next treatment line; 3) continuation of original treatment without palbociclib; 4) no BC therapy after the last dose of palbociclib, and still alive; 5) no BC therapy after the last dose of palbociclib, and died. Treatments administered after the last dose of palbociclib were examined (endocrine or chemotherapy).
Comorbidities were examined from 365 days prior to the first prescription claim for palbociclib to the last claim for palbociclib based on conditions included in the Charlson Comorbidity Index..$^{20}$ Considering that cardiovascular disease is the leading cause of death in Hungary and certain important cardiovascular diseases are not included in the Charlson Comorbidity Index, we added hypertension and arrhythmia to our analysis. Comorbidities were identified based on the occurrence of corresponding ICD-10 codes in inpatient care, or at least two outpatient occurrences of the same ICD-10 codes within 30 to 365 days.

Concomitant medications were examined from 45 days prior to the initiation of palbociclib to the last prescription claim for palbociclib, and classified according to the Anatomical Therapeutic Chemical (ATC) classification system as recommended by the WHO. ${ }^{21}$ The number of used drugs was defined as the number of used original drug compounds (5th-level ATC code: chemical substance). Polypharmacy was defined as the concomitant use of $>5$ drugs. The administration of drugs with a potential for drug-drug interactions (DDIs) with palbociclib was also examined. ${ }^{22}$ The proportion of patients receiving palliative oncology care modalities from 45 days prior to palbociclib initiation was also determined.

For all examined parameters, where crude numbers of any parameter were recorded below 10 , we indicated " $<10$ " as the Hungarian NHIF data protection law does not allow for the presentation of case numbers below 10 in a stratum.

Descriptive statistics was applied for the variables, and no formal hypothesis was tested. A pooled analysis was performed in the entire observation period, and separate analyses in the period of NPBR (May 1, 2017 to October 14, 2018) and regular reimbursement (October 15, 2018 to June 30, 2019). Furthermore, patient demographic and clinical characteristics as well as treatment patterns were compared between the two reimbursement periods. Mean age was compared by Welch-test, and proportions were compared by chi-squared test (with simulated $p$-values if any expected number was less than five). The significance level was set to $5 \%(0.05)$.

This non-interventional study was approved by the Hungarian Ethical Medical Research Council (licence number: OGYÉI/47600-5/2019) and has been carried out in accordance with the 1975 Declaration of Helsinki, as revised in 2000, and Good Clinical Practice guidelines.

Data collection and analysis were performed on anonymized and aggregated data. 


\section{Results}

A total of 962 female $\mathrm{ABC}$ patients were eligible for inclusion who received palbociclib therapy between May 1, 2017, and June 30, 2019, with a mean age of 60.6 (59.9-61.3) years. Eleven male patients received palbociclib during the study period, and 107 female patients received treatment for $\mathrm{ABC}$ other than bisphosphonates, AI or fulvestrant while on palbociclib. These patients were not included in our analysis. Demographic and clinical characteristics of patients are shown in Table 1.

Table 2 shows median follow-up times with 95\% CI for the whole patient population, the palbociclib plus AI, and palbociclib plus fulvestrant cohorts during the whole study period, and separately for the NPBR and regular reimbursement periods. Median follow-up was 8 months (95\% CI: 8-8) in the whole patient population, 14 months (95\% CI: 13-14) in the NBPR period, and 5 months $(95 \%$ CI: 5-6) in the regular reimbursement period.

The endocrine partner of palbociclib was an AI (letrozole, anastrozole, exemestane) in 399 patients (41\%), and fulvestrant in $563(59 \%)$ patients. A total of 849 patients (88\%) were postmenopausal and 113 patients $(12 \%)$ were pre/perimenopausal. A total of 733 patients $(76 \%)$ had metastatic disease, $884(92 \%)$ had recurrent $\mathrm{BC}$ and 78 ( $8 \%$ ) had de novo metastatic disease at the time of palbociclib initiation. A significantly higher proportion of patients had recurrent $\mathrm{BC}$ in the NPBR period compared to the regular reimbursement period in the total patient population as well as in the palbociclib plus AI group (96\% vs $88 \% ; \mathrm{p}<0.0001$ and $90 \%$ vs $74 \% ; \mathrm{p}<0.0001$, respectively). A total of 241 patients (33\%) had visceral and $449(61 \%)$ had bone-only metastases. During the NPBR period, the number of patients with visceral metastases including liver metastases was numerically higher, than in the regular reimbursement period (Supplementary Table 1).

The following treatment modalities were applied for patients who had received prior treatment for eBC: neoadjuvant chemotherapy ( $\mathrm{n}=95 ; 18 \%)$, adjuvant chemotherapy $(n=241 ; 47 \%)$, adjuvant endocrine therapy $(n=395 ; 76 \%)$, radiotherapy $(n=307 ; 59 \%)$, and/or surgery $(n=410 ; 79 \%)$. In the majority of cases, palbociclib was administered as first- or second-line therapy ( $51 \%$ and $28 \%$, respectively), and $19 \%$ of patients received palbociclib in third or further lines of treatment. Among patients $(n=453)$ with prior therapy for $\mathrm{ABC}$ before palbociclib initiation, 375 patients (83\%) had received endocrine therapy and 242 (53\%) received chemotherapy. The number of patients receiving palbociclib in third or further lines of therapy and of those receiving prior chemotherapy for $\mathrm{ABC}$ were numerically higher during the NPBR than the regular reimbursement period (Supplementary Table 1).

Among patients receiving palbociclib and an AI, the combination endocrine partner was letrozole in 359 patients $(90 \%)$, while $32(8 \%)$ received exemestane and $11(3 \%)$ received anastrozole. At the end of the observation period (June 30, 2019), palbociclib therapy was still ongoing in 270 patients $(68 \%)$ in the AI and in 245 patients $(44 \%)$ in the fulvestrant group. Sixty-nine (17\%) and $157(28 \%)$ patients were switched to either endocrine or chemotherapy from palbociclib plus AI and palbociclib plus fulvestrant, respectively, while $33(8 \%)$ and 115 $(20 \%)$ continued original treatment with an AI or fulvestrant without palbociclib. By June 30, 2019, 20 patients (5\%) in the palbociclib plus AI group and 38 patients $(7 \%)$ in the palbociclib plus fulvestrant groups had died after discontinuing ABC therapy (Table 3 ).

The starting dose of palbociclib was $125 \mathrm{mg} / \mathrm{d}$ in $98 \%$ of the patients ( $\mathrm{n}=941$ ), and it was $100 \mathrm{mg} / \mathrm{d}$ or $75 \mathrm{mg} / \mathrm{d}$ only in $2 \%$. Most patients $(n=643 ; 67 \%)$ did not require any dose reductions. A significantly higher proportion of patients were initiated on the $125 \mathrm{mg} / \mathrm{d}$ starting dose in the NPBR period than in the regular reimbursement period both in the palbociclib plus AI and palbociclib + fulvestrant groups (99\% vs $97 \%, \mathrm{p}=0.0070$ and $100 \%$ vs $95 \%$, $\mathrm{p}<0.0001$, respectively). The number of dose adjustments was numerically lower during the NPBR period compared to the regular reimbursement period (Table 3, Supplementary Table 2).

A total of 412 patients (43\%) had at least one nonmalignant comorbidity, the most common of which were hypertension $(n=315,33 \%)$ and diabetes mellitus $(n=100$, 10\%) (Table 4).

Fifty percent of patients $(n=479)$ received at least one drug with a potential for DDI with CDK4/6 inhibitors including palbociclib. The most common DDI medications included analgesics $(\mathrm{n}=257 ; 27 \%)$, antimicrobial drugs $(\mathrm{n}=195 ; 20 \%)$, medications for gastrointestinal conditions $(n=164 ; 17 \%)$ and for psychiatric disorders $(n=57 ; 6 \%)$. Known CYP3A4 inhibitors were administered in 97 patients (10\%) (Table 5).

The majority of patients $(n=555 ; 58 \%)$ received more than 5 concomitant medications during palbociclib therapy (Table 6). The most frequently used medications among patients with polypharmacy were bisphosphonates $(n=327$; 
Table I Patient Demographics and Clinical Characteristics of Patients Treated with Palbociclib During the Whole Study Period

\begin{tabular}{|c|c|c|c|}
\hline & PAL+Al & PAL+FUL & Overall \\
\hline Total patients, $\mathbf{n}$ & 399 & 563 & 962 \\
\hline \multicolumn{4}{|l|}{ Age at palbociclib initiation, years } \\
\hline Median & 61.0 & 63.0 & 62.0 \\
\hline Mean $(95 \% \mathrm{Cl})$ & $59.3(58.1-60.4)$ & $61.6(60.7-62.5)$ & $60.6(59.9-61.3)$ \\
\hline \multicolumn{4}{|l|}{ Menopause status, $\mathbf{n}(\%)$} \\
\hline Pre/perimenopausal & $68(17 \%)$ & $45(8 \%)$ & $113(12 \%)$ \\
\hline Postmenopausal & 331 (83\%) & 518 (92\%) & 849 (88\%) \\
\hline \multicolumn{4}{|l|}{ Stage at palbociclib initiation, $\mathbf{n}(\%)$} \\
\hline Locally advanced & $100(25 \%)$ & $129(23 \%)$ & $229(24 \%)$ \\
\hline Metastatic & 299 (75\%) & 434 (77\%) & 733 (76\%) \\
\hline \multicolumn{4}{|l|}{ Occurrence of stage IV breast cancer, n (\%) } \\
\hline “De novo” & 75 (19\%) & $<10(1 \%)$ & $78(8 \%)$ \\
\hline "Recurrent" & $324(81 \%)$ & 560 (99\%) & $884(92 \%)$ \\
\hline Metastatic sites, n (\%) & 299 (100\%) & $434(100 \%)$ & $733(100 \%)$ \\
\hline Bone only disease & 198 (66\%) & $25 \mathrm{I}(58 \%)$ & $449(61 \%)$ \\
\hline Visceral & $86(29 \%)$ & 155 (36\%) & $24 I(33 \%)$ \\
\hline Lung/pleura & 44 (5I\%) & 101 (65\%) & 145 (60\%) \\
\hline Liver & 47 (55\%) & 64 (4I\%) & III (46\%) \\
\hline CNS & $<10$ & $19(12 \%)$ & $28(12 \%)$ \\
\hline Ovarian & $<10$ & $<10$ & $<10$ \\
\hline Other & $10(3 \%)$ & $16(4 \%)$ & $26(4 \%)$ \\
\hline Unknown & $<10$ & $12(3 \%)$ & 17 (2\%) \\
\hline Prior therapy for eBC, $n$ (\%) & $176(100 \%)$ & $341(100 \%)$ & $517(100 \%)$ \\
\hline Neoadjuvant chemotherapy & $35(20 \%)$ & $60(17 \%)$ & $95(18 \%)$ \\
\hline Adjuvant chemotherapy & 72 (4I\%) & $169(50 \%)$ & $24 \mid(47 \%)$ \\
\hline Adjuvant endocrine therapy & 124 (70\%) & 271 (79\%) & 395 (76\%) \\
\hline Radiotherapy & 80 (45\%) & 227 (67\%) & 307 (59\%) \\
\hline Surgery & I 24 (70\%) & $286(84 \%)$ & 410 (79\%) \\
\hline \multicolumn{4}{|l|}{ Line of treatment at palbociclib initiation, $\mathbf{n}(\%)$} \\
\hline Ist line & $268(67 \%)$ & $220(39 \%)$ & $488(51 \%)$ \\
\hline 2nd line & $80(20 \%)$ & 192 (34\%) & $272(28 \%)$ \\
\hline 3rd line & $29(7 \%)$ & $90(16 \%)$ & $119(12 \%)$ \\
\hline 4th line & II (3\%) & $31(5 \%)$ & $42(5 \%)$ \\
\hline$>4$ th line & $<10$ & $15(3 \%)$ & $20(2 \%)$ \\
\hline Unknown & $<10$ & $15(3 \%)$ & $21(2 \%)$ \\
\hline Prior therapies for $A B C$ before palbociclib initiation, $\mathbf{n}(\%)$ & 125 & 328 & 453 \\
\hline Endocrine & $81(65 \%)$ & $294(90 \%)$ & $375(83 \%)$ \\
\hline Chemotherapy & $80(64 \%)$ & 162 (49\%) & $242(53 \%)$ \\
\hline Targeted (everolimus) & $<10$ & $32(10 \%)$ & $40(9 \%)$ \\
\hline Other & $<10$ & $<10$ & 12 (3\%) \\
\hline
\end{tabular}

Abbreviations: ABC, advanced breast cancer; CNS, central nervous system; eBC, early breast cancer; PAL+AI, palbociclib plus aromatase inhibitor; PAL+FUL, palbociclib plus fulvestrant.

$59 \%)$, vitamin $D$ and its analogs $(n=265 ; 48 \%)$, proton pump inhibitors $(\mathrm{n}=217 ; 39 \%)$, selective $\beta$-blockers $(n=207 ; 37 \%)$, calcium $(n=166 ; 30 \%)$, and opioids $(\mathrm{n}=160 ; 29 \%)$ (Supplementary Table 3).
A total of 498 patients (52\%) received palliative oncology care which involved interventions aimed at improving quality of life, physical and mental well-being. The most frequent treatment modalities were analgesia in 428 
Table 2 Median Follow-Up Times $(95 \% \mathrm{Cl})$ for the Whole Patient Population, the Palbociclib Plus Al, and Palbociclib Plus Fulvestrant Cohorts During the Whole Study Period (May I, 2017-June 30, 2019), and Separately for the NPBR (May I, 2017-October I, 2018) and Regular Reimbursement Periods (October 15, 2018-June 30, 2019)

\begin{tabular}{|l|c|c|c|}
\hline \multirow{2}{*}{} & \multicolumn{3}{|c|}{ Median Follow-Up, Months (95\% Cl) } \\
\cline { 2 - 4 } & NPBR & RR & Total \\
\hline PAL+AI & $14(13-15)$ & $6(5-6)$ & $8(8-9)$ \\
PAL+FUL & $14(13-14)$ & $5(4-6)$ & $8(8-9)$ \\
Total & $14(13-14)$ & $5(5-6)$ & $8(8-8)$ \\
\hline
\end{tabular}

Abbreviations: $\mathrm{Cl}$, confidence interval. NPBR, named-patient based reimbursement; PAL+Al, palbociclib plus aromatase inhibitor; $P A L+F U L$, palbociclib plus fulvestrant; RR, regular reimbursement.

(44\%), therapeutic gymnastics/rehabilitation in $79(8 \%)$, and psychological/psychiatric care in 30 patients $(3 \%)$. Depression or anxiety occurred in 92 patients $(10 \%)$ (Table 7).

\section{Discussion}

This retrospective study is the first to report data on the real-world use of palbociclib from a Central-Eastern European country, describing patient demographics and clinical characteristics as well as disease and treatmentrelated factors in a large population of 962 patients with advanced HR+/HER2- ABC. Since the regulatory approval of palbociclib, a number of real-world studies have examined the use of palbociclib in routine clinical practice. The Ibrance Real World Insights Study (IRIS) was initiated to complement clinical trial data, and results from the United States, Argentina, and Germany consistently support the effectiveness and tolerability of palbociclib in unselected patient populations. ${ }^{23-25}$ Apart from the IRIS program, RENATA, POLARIS, and an Italian retrospective study also provided real-world data on palbociclib prescribing and treatment patterns in routine clinical practice, and the Italian PALPract study evaluated the efficacy and safety of palbociclib combined with either letrozole or fulvestrant in a real-world setting including patients from 14 cancer centres. $^{26-30}$ Our retrospective analysis fits well into the growing body of real-world evidence with palbociclib and complements recent observations from other countries.

The demographic and clinical characteristics of our patient population were largely consistent with the IRIS cohorts in terms of mean age, menopausal status, disease

Table 3 Treatment Patterns of Patients Receiving Palbociclib Therapy During the Whole Study Period

\begin{tabular}{|c|c|c|c|}
\hline & PAL+Al & PAL+FUL & Overall \\
\hline Total patients, $\mathbf{n}$ & 399 & 563 & 962 \\
\hline \multicolumn{4}{|l|}{ Combination endocrine partner*, n (\%) } \\
\hline Letrozole & $359(90 \%)$ & - & $359(37 \%)$ \\
\hline Anastrozole & II (3\%) & - & II (I\%) \\
\hline Exemestane & $32(8 \%)$ & - & $32(3 \%)$ \\
\hline Fulvestrant & - & $563(100 \%)$ & $563(59 \%)$ \\
\hline \multicolumn{4}{|c|}{ Follow-up of palbociclib treated patients on 30 June 2019} \\
\hline Palbociclib treatment is ongoing & $270(68 \%)$ & $245(44 \%)$ & $515(54 \%)$ \\
\hline Switch to next therapy & $69(17 \%)$ & $157(28 \%)$ & $226(23 \%)$ \\
\hline Continuation of endocrine therapy without palbociclib & $33(8 \%)$ & II 5 (20\%) & $148(15 \%)$ \\
\hline No therapy for breast cancer and alive & $<10$ & $<10$ & $15(2 \%)$ \\
\hline No therapy for breast cancer and died & $20(5 \%)$ & $38(7 \%)$ & $58(6 \%)$ \\
\hline Next therapy following palbociclib* & $69(100 \%)$ & $157(100 \%)$ & $226(100 \%)$ \\
\hline Endocrine therapy & $40(58 \%)$ & $65(41 \%)$ & $105(46 \%)$ \\
\hline Chemotherapy & $30(43 \%)$ & $93(59 \%)$ & $123(54 \%)$ \\
\hline Starting dose: 125 mg, n (\%) & 391 (98\%) & $550(98 \%)$ & 941 (98\%) \\
\hline No dose reduction & $266(68 \%)$ & $377(69 \%)$ & $643(68 \%)$ \\
\hline Only one prescription claim & $20(5 \%)$ & $47(9 \%)$ & $67(7 \%)$ \\
\hline Dose reduced to $100 \mathrm{mg}$ & $78(20 \%)$ & $84(15 \%)$ & $162(17 \%)$ \\
\hline Dose reduced to $75 \mathrm{mg}$ & 27 (7\%) & 42 (7\%) & 69 (7\%) \\
\hline
\end{tabular}

Notes: $*$ The results are reported in the pre-specified groups, the non-substantial numerical differences can not be commented as data privacy low applied by NHIF does not allow reporting of any groups $<10$ patients.

Abbreviations: PAL+Al, palbociclib plus aromatase inhibitor; PAL+FUL, palbociclib plus fulvestrant. 
Table 4 Most Common Comorbidities of Patients Treated with Palbociclib

\begin{tabular}{|l|c|c|c|}
\hline & PAL+Al & PAL+FUL & Overall \\
\hline All patients, $\mathbf{n}$ & $\mathbf{3 9 9}$ & $\mathbf{5 6 3}$ & $\mathbf{9 6 2}$ \\
\hline Patients without reported comorbidity, $\mathbf{n}(\%)$ & $236(59 \%)$ & $314(56 \%)$ & $550(57 \%)$ \\
\hline Patients with reported comorbidity, $\mathbf{n}(\%)$ & $163(41 \%)$ & $249(44 \%)$ & $412(43 \%)$ \\
\hline Hypertension & $133(33 \%)$ & $182(32 \%)$ & $315(33 \%)$ \\
Diabetes & $35(9 \%)$ & $65(12 \%)$ & $100(10 \%)$ \\
Ischaemic heart disease & $27(7 \%)$ & $40(7 \%)$ & $67(7 \%)$ \\
Pulmonary disease (lower tract) & $25(6 \%)$ & $42(7 \%)$ & $67(7 \%)$ \\
Arrhythmia (including AF) & $22(6 \%)$ & $22(4 \%)$ & $44(5 \%)$ \\
Thromboembolism & $19(5 \%)$ & $26(5 \%)$ & $45(5 \%)$ \\
Cerebrovascular accident & $<10$ & $26(5 \%)$ & $34(4 \%)$ \\
Peripheral vascular disease & $11(3 \%)$ & $17(3 \%)$ & $28(3 \%)$ \\
Congestive heart failure & $<10$ & $10(2 \%)$ & $19(2 \%)$ \\
\hline
\end{tabular}

Abbreviations: AF, atrial fibrillation; PAL+Al, palbociclib plus aromatase inhibitor; PAL+FUL, palbociclib plus fulvestrant.

Table 5 Concomitant Medications with a Potential for Drug-Drug Interactions for CDK4/6 Inhibitors Among Patients Treated with Palbociclib

\begin{tabular}{|c|c|c|c|}
\hline $\begin{array}{l}\text { Concomitant Medications Utilized Within the Period } \\
\text { Starting } 45 \text { Days Prior to First Prescription of Palbociclib to } \\
\text { the Last Prescription Day of Palbociclib }\end{array}$ & PAL+Al & PAL+FUL & Overall \\
\hline Total patients, $\mathbf{n}$ & 399 & 563 & 962 \\
\hline Total patients without potential DDIs, n (\%) & $201(50 \%)$ & $282(50 \%)$ & $483(50 \%)$ \\
\hline Total patients with potential DDIs, n (\%) & $198(50 \%)$ & $281(50 \%)$ & $479(50 \%)$ \\
\hline $\begin{array}{l}\text { Analgesic drugs, } \mathbf{n}(\%) \\
\text { (eg methadone, tramadol) }\end{array}$ & $110(28 \%)$ & 147 (26\%) & $257(27 \%)$ \\
\hline $\begin{array}{l}\text { Antimicrobial agents, } \mathbf{n}(\%) \\
\text { (eg ciprofloxacin, fluconazole) }\end{array}$ & $80(20 \%)$ & II 5 (20\%) & $195(20 \%)$ \\
\hline $\begin{array}{l}\text { Gastrointestinal disorders drugs, } \mathbf{n} \text { (\%) } \\
\text { (eg esomeprazole, ondansetron) }\end{array}$ & $68(17 \%)$ & $96(17 \%)$ & $164(17 \%)$ \\
\hline $\begin{array}{l}\text { CYP3A inhibitors, } \mathbf{n} \text { (\%) } \\
\text { (eg azithromycin, clarithromycin) }\end{array}$ & $4 I(10 \%)$ & $56(10 \%)$ & $97(10 \%)$ \\
\hline $\begin{array}{l}\text { Psychiatric drugs, n (\%) } \\
\text { (eg citalopram, paroxetine) }\end{array}$ & $18(5 \%)$ & $39(7 \%)$ & $57(6 \%)$ \\
\hline $\begin{array}{l}\text { P-gp inhibitors, n (\%) } \\
\text { (eg ketoconazole, itraconazole) }\end{array}$ & $<10$ & $<10$ & $10(1 \%)$ \\
\hline
\end{tabular}

Abbreviations: DDI, drug-drug interaction; PAL+AI, palbociclib plus aromatase inhibitor; PAL+FUL, palbociclib plus fulvestrant.

stage at the time of palbociclib initiation, rate of recurrent and de novo metastatic breast cancer, and distribution of prior therapies for eBC. The vast majority of our patients $(98 \%)$ were started on $125 \mathrm{mg} /$ day dose of palbociclib according to EMA label recommendations, and $90 \%$ of those receiving palbociclib plus an AI were treated with letrozole correspondently the PALOMA-1 and PALOMA2 study protocols. These results show that Hungarian physicians strictly adhere to evidence-based recommendations and experience from clinical trials both during early access program and regular reimbursement. The majority of patients $(\sim 70 \%)$ did not require dose reductions, which shows that palbociclib was well tolerated and well managed in this real-world patient population and suggests that there is no rationale for initiating palbociclib at a reduced dose. Our results are very similar to the registrational PALOMA-2 and PALOMA-3 trials, where dose reductions were required in $36 \%$ and $34 \%$ of 
Table 6 Polypharmacy in Patients Treated with Palbociclib - 45 Days Prior to and/or During Palbociclib Initiation, ACT5 Level

\begin{tabular}{|l|c|c|c|}
\hline Number of ATC5 Groups & PAL+AI & PAL+FUL & Overall \\
\hline Total patients, $\boldsymbol{n}(\%)$ & $\mathbf{3 9 9}(\mathbf{1 0 0 \% )}$ & $\mathbf{5 6 3}(\mathbf{1 0 0 \% )}$ & $\mathbf{9 6 2}(\mathbf{1 0 0 \% )}$ \\
$0-1$ & $29(7 \%)$ & $46(8 \%)$ & $75(8 \%)$ \\
$2-5$ & $148(37 \%)$ & $184(33 \%)$ & $332(35 \%)$ \\
$6-8$ & $102(26 \%)$ & $135(24 \%)$ & $237(25 \%)$ \\
$>8$ & $120(30 \%)$ & $198(35 \%)$ & $318(33 \%)$ \\
$>\mathbf{5}$ (polypharmacy) & $222(56 \%)$ & $333(59 \%)$ & $555(58 \%)$ \\
\hline
\end{tabular}

Abbreviations: PAL+AI, palbociclib plus aromatase inhibitor; PAL+FUL, palbociclib plus fulvestrant.

patients, respectively. ${ }^{9,10}$ The majority of recent real-world studies have also reported consistently lower dose reduction rates than the PALOMA studies, which provides reassurance regarding the good tolerability of palbociclib in unselected patient populations and the benefits of patient and staff education. ${ }^{24-27,31}$

In our study, more than $40 \%$ of patients receiving palbociclib had at least one non-malignant comorbidity, mostly hypertension and diabetes. This is in line with observations from other studies which reported cardiovascular conditions and/or diabetes among the most common comorbidities of patients with BC. ${ }^{17,18,32-35}$ One of the strengths of our nationwide study lies in the fact that our study population included all patients with $\mathrm{ABC}$ who were receiving palbociclib in Hungary during the study period. Furthermore, apart from examining the prevalence of comorbidities in our overall patient population, we also provided separate and detailed information on patients receiving palbociclib with aromatase inhibitors and those treated with palbociclib and fulvestrant. The potential clinical relevance of differences in the prevalence of comorbidities between the two groups requires further investigations. Our findings complement previous observations regarding the comorbidity profile of patients with $A B C$ in the real world. Since several reports have pointed out the association between comorbidities and survival as well as quality of life in $\mathrm{BC}$ patients, the better understanding of comorbidity profiles among patients with $\mathrm{ABC}$ is of utmost importance. . $^{32,33,36-38}$

$\mathrm{HR}+$ breast cancer is primarily a disease of the elderly, who are also known to have a higher incidence of polypharmacy due to chronic diseases which require multiple medications. ${ }^{39,40}$ Elderly patients are also at an increased risk of adverse events resulting from DDIs due to metabolic changes and reduced drug clearance associated with ageing. ${ }^{40}$ Polypharmacy and DDIs are a significant concern in oncology which may limit the therapeutic value of anticancer therapies including CDK4/6 inhibitors. Palbociclib is a substrate and weak inhibitor of CYP3A, and a moderate substrate of P-glycoprotein; therefore, the co-administration of palbociclib with CYP3A4 inhibitors or CYP3A4 inducers requires caution in routine clinical practice. $^{15}$ To our knowledge, our study was the first to investigate the incidence of polypharmacy and DDIs among patients treated with palbociclib in the real world. We found that nearly $60 \%$ of patients were taking at least 5 concomitant medications, even though over-the-counter drugs were not included in the analysis. Our findings highlight that polypharmacy and DDIs affect a clinically relevant proportion of $\mathrm{ABC}$ patients treated with palbociclib in

Table 7 Palliative Oncology Care in Patients Treated with Palbociclib

\begin{tabular}{|c|c|c|c|}
\hline $\begin{array}{l}\text { Palliative Oncology Care Provided Within } 45 \text { Days Prior to } \\
\text { the First Prescription of Palbociclib to the Last Prescription } \\
\text { Day of Palbociclib }\end{array}$ & PAL+AI & PAL+FUL & Overall \\
\hline Total patients, $\mathbf{n}$ & 399 & 563 & 962 \\
\hline Total patients without palliative care, $\mathbf{n}(\%)$ & $194(49 \%)$ & $270(48 \%)$ & $464(48 \%)$ \\
\hline Total patients with Palliative care, n (\%) & $205(51 \%)$ & $293(52 \%)$ & $498(52 \%)$ \\
\hline Analgesia total, n (\%) & 175 (44\%) & $253(45 \%)$ & $428(44 \%)$ \\
\hline Opioids & $124(3 \mid \%)$ & $170(30 \%)$ & $294(31 \%)$ \\
\hline NSAIDs & 101 (25\%) & $159(28 \%)$ & $260(27 \%)$ \\
\hline Occurrence of depression/anxiety total, n (\%) & $39(10 \%)$ & $53(9 \%)$ & $92(10 \%)$ \\
\hline Therapeutic gymnastics/rehabilitation total, n (\%) & $4 \mathrm{l}(10 \%)$ & $38(7 \%)$ & $79(8 \%)$ \\
\hline Psychological/psychiatric care total, n (\%) & $14(4 \%)$ & $16(3 \%)$ & $30(3 \%)$ \\
\hline Lymphoedema treatment total, $\mathrm{n}(\%)$ & $<10$ & $<10$ & $<10$ \\
\hline
\end{tabular}

Abbreviations: NSAID, non-steroidal anti-inflammatory drug; PAL+AI, palbociclib plus aromatase inhibitor; PAL+FUL, palbociclib plus fulvestrant. 
routine clinical practice. However, the high proportion of patients receiving DDI medications in our study also suggest that the co-administration of palbociclib with these drugs is seemed to be feasible with the continuous and careful evaluation of risks and benefits.

At the end of the observation period, palbociclib therapy was still ongoing in $68 \%$ of patients receiving palbociclib plus AI, and in $44 \%$ of those receiving palbociclib with fulvestrant. Sixty-nine and 157 patients received next therapy after palbociclib in the PAL+AI and the PAL+FUL groups, which was chemotherapy in $43 \%$ and $59 \%$ of patient retrospectively. The high rate of chemotherapy use for the treatment of $\mathrm{HR}+$ patients reveals the need for stricter adherence to guidelines in terms of sequencing endocrine-based therapy.

When comparing our findings between the two different reimbursement periods (early access vs regular reimbursement), we found that the proportion of patients with recurrent $\mathrm{BC}$ vs those with de novo metastatic disease was significantly higher in the NPBR vs regular reimbursement period in the whole patient population and in the palbociclib plus AI group. A possible explanation for this difference may be that in the NPBR period, treating physicians may have had concerns regarding the initiation of palbociclib therapy for patients with de novo metastatic disease due to the lack of experience. By the time palbociclib became available under regular reimbursement, clinicians had gained sufficient experience with the use of palbociclib and may have become more confident regarding its use in de novo metastatic disease. The proportion of patients started on the palbociclib $125 \mathrm{mg} / \mathrm{d}$ dose was also significantly higher in the NPBR vs regular reimbursement period in the whole patient population. In fact, the vast majority of patients were started on the recommended $125 \mathrm{mg} / \mathrm{d}$ dose in both treatment groups during both study periods. The statistically significant difference between the NPBR and RR periods reflects very small differences in percentages $(100 \%$ vs $96 \%)$ and may not be considered clinically meaningful in actual clinical practice. In addition, we found slight numerical differences in terms of the proportion of patients with visceral disease, line of palbociclib therapy, prior chemotherapy, and the incidence of dose reductions. These differences may be explained by the approval process of NPBR causing delays in treatment initiation and the administrative burden of prescribing reduced doses which was no longer the case after the regular reimbursement of palbociclib in Hungary, and by the gradual change in the ingrained clinical practice of using monotherapy for HR+/HER2- ABC patients. Nevertheless, our findings show that the named-patientbased reimbursement system allowed for the appropriate and timely initiation of palbociclib therapy even before regular reimbursement.

Our study has certain limitations. First, the follow-up time in our study was not long enough for the assessment of effectiveness outcomes, such as overall survival or PFS. A further analysis is planned to examine palbociclib treatment outcomes with a longer follow-up period. Second, the NHIF database does not contain any information on laboratory parameters, vital signs or prognostic factors such as ECOG performance status, and it is not suitable for the analysis of therapeutic response. Third, we could not exclude the potential underreporting of comorbidities and/or inaccuracies in reporting. Fourth, healthcare services outside the state-financed system could not be captured and thus were not included in the analysis.

\section{Conclusions}

This nationwide retrospective study conducted in Hungary provides further insights into patient characteristics and real-world treatment patterns on the use of palbociclib in combination with endocrine therapy in patients with HR +/HER2 - advanced breast cancer. Our findings complement previous results in a large patient population and provide reassurance regarding the use of palbociclib in routine clinical practice.

\section{Acknowledgments}

We would like to thank our colleagues at RxTarget Ltd. for their invaluable support in statistical analysis, Zsófia Barcza of Syntesia Medical Communications Ltd. for medical writing support, and the National Health Insurance Fund for providing comprehensive datasets for our analysis.

\section{Funding}

This study was sponsored by Pfizer Ltd. Medical writing was provided by Syntesia Medical Communications Ltd., and was funded by Pfizer Ltd. Data collection was funded by Pfizer Ltd.

\section{Disclosure}

Katalin Boér has been a consultant or on the advisory board for Eli Lilly, Novartis, Pfizer, and Roche. György Rokszin and Zsolt Abonyi-Tóth are employees of RxTarget Ltd., where their contribution to this study analysis was financially compensated by Pfizer Hungary Ltd. 
During the writing of the manuscript Csenge Földesi was employee of and owned stock in Pfizer Inc. Magdolna Dank has been a consultant or on the advisory board for Eli Lilly, Novartis, Pfizer and Roche. Gábor Rubovszky had consulting or lecturer fee from Amgen, Eli Lilly, MDS, Novartis, Pfizer, Roche, SWIXX. The authors report no other conflicts of interest in this work.

\section{References}

1. Surveillance, Epidemiology, and End Results (SEER) Program. Cancer Stat Facts: Female Breast Cancer Subtypes. Available from: https://seer.cancer.gov/statfacts $/ \mathrm{html} /$ breast-subtypes.html. Accessed August 24, 2020].

2. Howlader N, Altekruse SF, Li CI, et al. US incidence of breast cancer subtypes defined by joint hormone receptor and HER2 status. $J$ Natl Cancer Inst. 2014;106(5):dju055. doi:10.1093/jnci/dju055

3. Rocca A, Farolfi A, Bravaccini S, Schirone A, Amadori D. Palbociclib (pd 0332991): targeting the cell cycle machinery in breast cancer. Expert Opin Pharmacother. 2014;15(3):407-420.

4. National Institute for Health and Care Excellence (NICE) Managing advanced breast cancer. 2017. Available from: https://pathways.nice.org. uk/pathways/advanced-breast-cancer\#path=view\%3A/pathways/ advanced-breast-cancer/managing-advanced-breast-cancer.xml\&content= view-node\%3Anodes-hrpos-and-her2neg. Accessed August 6, 2020].

5. Sini V, Cinieri S, Conte $P$, et al. Endocrine therapy in post-menopausal women with metastatic breast cancer: from literature and guidelines to clinical practice. Crit Rev Oncol Hematol. 2016;100:57-68. doi:10.1016/j.critrevonc.2016.02.008

6. Glück S. Consequences of the Convergence of Multiple Alternate Pathways on the Estrogen Receptor in the Treatment of Metastatic Breast Cancer. Clin Breast Cancer. 2017;17(2):79-90.

7. Cardoso F, Paluch-Shimon S, Senkus E, et al. 5th ESO-ESMO international consensus guidelines for advanced breast cancer (ABC 5). Ann Oncol. 2020;31(12):1623-1649. doi:10.1016/j.annonc.20 20.09.010

8. Bayraktar S, Batoo S, Al-Hattab E, Basu S, Okuno S, Glück S. Future perspectives and challenges with CDK4/6 inhibitors in hormone receptor-positive metastatic breast cancer. Future Oncol. 2020;16 (32):2661-2672.

9. Piezzo M, Chiodini P, Riemma M, et al. Progression-free survival and overall survival of CDK $4 / 6$ inhibitors plus endocrine therapy in metastatic breast cancer: a systematic review and meta-analysis. Int J Mol Sci. 2020;21(17):6400. doi:10.3390/ijms21176400

10. Finn RS, Crown JP, Lang I, et al. The cyclin-dependent kinase $4 / 6$ inhibitor palbociclib in combination with letrozole versus letrozole alone as first-line treatment of oestrogen receptor-positive, HER2-negative, advanced breast cancer (PALOMA-1/TRIO-18): a randomised Phase 2 study. The Lancet Oncology. 2015;16 (1):25-35. doi:10.1016/S1470-2045(14)71159-3

11. Finn RS, Martin M, Rugo HS, et al. Palbociclib and letrozole in advanced breast cancer. N. Engl. J. Med. 2016;375(20):1925-1936.

12. Cristofanilli M, Turner NC, Bondarenko I, et al. Fulvestrant plus palbociclib versus fulvestrant plus placebo for treatment of hormone-receptor-positive, HER2-negative metastatic breast cancer that progressed on previous endocrine therapy (PALOMA-3): final analysis of the multicentre, double-blind, Phase 3 randomised controlled trial. Lancet Oncol. 2016;17(4):425-439.

13. Rugo HS, Finn RS, Diéras V, et al. Palbociclib plus letrozole as first-line therapy in estrogen receptor-positive/human epidermal growth factor receptor 2-negative advanced breast cancer with extended follow-up. Breast Cancer Res Treat. 2019;174 (3):719-729. doi:10.1007/s10549-018-05125-4
14. Turner NC, Ro J, André F, et al. Palbociclib in hormone-receptorpositive advanced breast cancer. $N$ Engl $J$ Med. 2015;373 (3):209-219. doi:10.1056/NEJMoa1505270

15. European Medicines Agency. Palbociclib Summary of Product Characteristics. 2020. Available from: https://www.ema.europa.eu/ en/documents/product-information/ibrance-epar-productinformation_en.pdf. Accessed May 17, 2021].

16. Inotai A, Csanádi M, Harsányi A, Németh B. Drug policy in hungary. Value Health Reg Issues. 2017;13:16-22. doi:10.1016/j. vhri.2017.06.003

17. Arpino G, Michelotti A, Truini M, et al. Demographic, tumor and clinical features of clinical trials versus clinical practice patients with HER2-positive early breast cancer: results of a prospective study. J Cancer Res Clin Oncol. 2016;142(3):669-678.

18. De Placido S, Giuliano M, Schettini F, et al. Human epidermal growth factor receptor 2 dual blockade with trastuzumab and pertuzumab in real life: Italian clinical practice versus the CLEOPATRA trial results. Breast. 2018;38:86-91.

19. Klonoff DC. The expanding role of real-world evidence trials in health care decision making. J Diabetes Sci Technol. 2020;14(1):174-179.

20. Charlson ME, Pompei P, Ales KL, MacKenzie CR. A new method of classifying prognostic comorbidity in longitudinal studies: development and validation. J Chronic Dis. 1987;40:373-383.

21. WHO Collaborating Centre for drug statistics methodology, Oslo, Norway. 2011. Available from: http://www.whocc.no. Accessed August 5, 2020.

22. Fogli S, Del Re M, Curigliano G, van Schaik RH, Lancellotti P, Danesi R. Drug-drug interactions in breast cancer patients treated with CDK4/6 inhibitors. Cancer Treat Rev. 2019;74:21-28.

23. Waller J, Mitra D, Mycock K, et al. Real-World Treatment Patterns and Clinical Outcomes in Patients Receiving Palbociclib for Hormone Receptor-Positive, Human Epidermal Growth Factor Receptor 2-Negative Advanced or Metastatic Breast Cancer in Argentina: the IRIS Study. J Glob Oncol. 2019;5:JGO1800239. doi:10.1200/JGO.18.00239

24. Taylor-Stokes G, Mitra D, Waller J, Gibson K, Milligan G, Iyer S. Treatment patterns and clinical outcomes among patients receiving palbociclib in combination with an aromatase inhibitor or fulvestrant for HR+/HER2-negative advanced/metastatic breast cancer in real-world settings in the US: results from the IRIS study. Breast. 2019;43:22-27. doi:10.1016/j.breast.2018.10.009

25. Taylor-Stokes G, Zhan L, Mycock KL, Miligan G, Ghale A, Mitra D. Real world treatment patterns and clinical outcomes associated with palbociclib combination therapy in Germany: results from the IRIS Study. Ann Oncolog. 2020;31(suppl_2):S62-S82.

26. Petracci F, Abuin GG, Pini A, Chacón M. RENATA study-Latin American prospective experience: clinical outcome of patients treated with palbociclib in hormone receptor-positive metastatic breast cancer-real-world use. Ecancermedicalscience. 2020;14:1058. doi:10.3332/ecancer.2020.1058

27. Pizzuti L, Giordano A, Michelotti A, et al. Palbociclib plus endocrine therapy in HER2 negative, hormonal receptor-positive, advanced breast cancer: a real-world experience. $J$ Cell Physiol. 2019;234(6):7708-7717.

28. Tripathy D, Blum JL, Rocque GB, et al. POLARIS: a prospective, multicenter, noninterventional study assessing palbociclib in hormone receptor-positive advanced breast cancer [published online ahead of print, 2020 Aug 13]. Future Oncol. 2020. doi:10.2217/fon-2020-0573

29. Blum JL, McCune SL, Salken MA, et al. First report of real-world patient characteristics and treatment patterns from POLARIS: palbociclib in hormone receptor-positive ( $\mathrm{HR}+$ ) advanced breast cancer: a prospective, multicenter, noninterventional study. Ann Oncolog. 2018;29(suppl_8):viii90-viii121. doi:10.1093/annonc/mdy272

30. Fabi A, Russillo M, Ciccarese M, et al. Real-world evidence of efficacy and activity of palbociclib plus endocrine therapy and post-progression treatments in HR+/HER2- metastatic breast cancer patients: the PALPract study. Cancer Res. 2020;80(4 Supplement): P5-11-18. doi:10.1158/1538-7445.SABCS19-P5-11-18 
31. Gong J, Cho M, Yu KW, Waisman J, Yuan Y, Mortimer J. A single institution experience with palbociclib toxicity requiring dose modifications. Breast Cancer Res Treat. 2018;168(2):381-387.

32. Fu MR, Axelrod D, Guth AA, et al. Comorbidities and Quality of Life among Breast Cancer Survivors: a Prospective Study. J Pers Med. 2015;5(3):229-242.

33. Yancik R, Wesley MN, Ries LA, Havlik RJ, Edwards BK, Yates JW. Effect of age and comorbidity in postmenopausal breast cancer patients aged 55 years and older. JAMA. 2001;285(7):885-892.

34. Sharma N, Narayan S, Sharma R, Kapoor A, Kumar N, Nirban R. Association of comorbidities with breast cancer: an observational study. Trop J Medl Res. 2015;19:168-171.

35. Ng HS, Vitry A, Koczwara B, Roder D, McBride ML. Patterns of comorbidities in women with breast cancer: a Canadian population-based study. Cancer Causes Control. 2019;30 (9):931-941.
36. Wesley MN, Ries LA, Havlik RJ, Havlik RJ, Edwards BK, Yates JW. Effect of age and comorbidity in postmenopausal breast cancer patients aged 55 years and older. JAMA. 2001;285(7):885-892.

37. Ewertz M, Land LH, Dalton SO, Cronin-Fenton D, Jensen MB. Influence of specific comorbidities on survival after early-stage breast cancer. Acta Oncol. 2018;57(1):129-134.

38. Braithwaite D, Moore DH, Satariano WA, et al. Prognostic impact of comorbidity among long-term breast cancer survivors: results from the LACE study. Cancer Epidemiol Biomarkers Prev. 2012;21 (7):1115-1125.

39. Charlesworth CJ, Smit E, Lee DS, Alramadhan F, Odden MC. Polypharmacy Among Adults Aged 65 Years and Older in the United States: 1988-2010. J Gerontol a Biol Sci Med Sci. 2015;70 (8):989-995. doi:10.1093/gerona/glv013

40. Dagli RJ, Sharma A. Polypharmacy: a global risk factor for elderly people. J Int Oral Health. 2014;6(6):i-ii.

\section{Publish your work in this journal}

OncoTargets and Therapy is an international, peer-reviewed, open access journal focusing on the pathological basis of all cancers, potential targets for therapy and treatment protocols employed to improve the management of cancer patients. The journal also focuses on the impact of management programs and new therapeutic agents and protocols on patient perspectives such as quality of life, adherence and satisfaction. The manuscript management system is completely online and includes a very quick and fair peer-review system, which is all easy to use. Visit http://www.dovepress.com/ testimonials.php to read real quotes from published authors. 